\title{
SOME EXAMPLES IN TOPOLOGY
}

\author{
BY \\ S. P. FRANKLIN AND M. RAJAGOPALAN
}

\begin{abstract}
. $\$ 1$ is concerned with variations on the theme of an ordinal compactification of the integers. Several applications are found, yielding, for instance, an example previously known only modulo the continuum hypothesis, and a counterexample to a published assertion.

$\$ 2$ is concerned with zero-one sequences and $\$ 3$ with spaces built from sequential fans. Of two old problems of Cech, one is solved and one partly solved.

Since the sections are more or less independent, each will have its own introduction. Sequential spaces form the connecting thread, although not all the examples are concerned with them.
\end{abstract}

1. Spaces such as $\beta N$ and $I^{N_{1}}$ provide ready examples of separable compact Hausdorff spaces which are not sequential $\left({ }^{1}\right)$. But these are of "large" cardinality, i.e. $2^{\mathfrak{c}}$. The space $\omega_{1}+1$ with the order topology is a nonsequential, compact Hausdorff space of "small" cardinality, i.e. $\aleph_{1}$, but, unfortunately, it is not separable. This leads one naturally to ask if there is a nonsequential, but separable, compact Hausdorff space of small cardinality. Such a space can be produced simply by conjoining known theorems as follows.

Magill [M, Theorem 2.1] showed that if any Hausdorff space $K$ is the continuous image of $\beta X \backslash X$, with $X$ locally compact Hausdorff, then there is a compactification $\gamma X$ of $X$ with $\gamma X \backslash X$ homeomorphic to $K$. Parovicenko [P, Theorem 1] proved that every compact Hausdorff space of weight $\leqq \boldsymbol{N}_{1}$ is the continuous image of $\beta N \mid N$. From these results one obtains

EXAMPLE 1.1. There is a compactification $\gamma \boldsymbol{N}$ of $\boldsymbol{N}$ with $\gamma \boldsymbol{N} \backslash \boldsymbol{N}$ homeomorphic to $\omega_{1}+1$, and hence there is a nonsequential, but separable, compact Hausdorff space of cardinality $\boldsymbol{\aleph}_{1}$.

By providing a specific construction of the space $\gamma \boldsymbol{N}$, which is done below, we can assure (modulo the continuum hypothesis $(\mathrm{CH})$ ) that no sequence in $N$ converges to $\omega_{1} \in \gamma N$. Then by modifying the topology of $\gamma N$ at the point $\omega_{1}$, we get

EXAMPLE 1.2. (CH) There is a sequentially compact, Hausdorff $c$-space $\left({ }^{2}\right)$ which

Received by the editors May 18, 1970.

AMS 1970 subject classifications. Primary 54G20, 54D55, 54D35, 54B17; Secondary 54A10, 54A25, 54D15, 54D20, 22A05.

Key words and phrases. Compactification, sequential, $c$-space, metacompact, sequentially compact, coreflection, topological group, Fréchet space, homogeneous.

(') A set is sequentially open if no sequence outside converges to a point inside. A sequential space is one in which every sequentially open set is open.

${ }^{2}$ ) A c-space is one in which the closure of each set is the union of the closures of its countable subsets. 
is not sequential. This corrects a mistake of the first author, showing that the proposition in $\left[\mathrm{F}_{4}\right]$ is false. This space, however, is not regular. The existence of such an example which is regular is still an open question.

Several fairly complicated examples have been given of separable and normal but nonparacompact spaces (see $\left[\mathrm{R}_{1}\right],[\mathrm{McA}]$ ). Having $\gamma N$ in hand, the simple expedient of omitting the point $\omega_{1}$ from $\gamma \boldsymbol{N}$ yields

EXAMPLE 1.3. $\gamma N \backslash\left\{\omega_{1}\right\}$ is a first-countable, locally compact space of cardinality $\aleph_{1}$ which is normal and separable, but not metacompact (and hence not paracompact). $\mathrm{CH}$ is not needed here.

Example 1.3 is similar to that of $\left[R_{1}\right]$.

In response to a question of $\mathrm{E}$. Michael, Mrs. Rudin $\left[\mathrm{R}_{2}, \S 1\right]$ constructed, modulo $\mathrm{CH}$, a normal, sequentially compact, but noncompact space with a separable, metric, locally compact, dense subset. Again modulo $\mathrm{CH}$, we can construct such a space with even nicer properties (i.e. first-countability, local compactness) as well as a simpler proof.

EXAMPLE 1.4. (CH) $\gamma N$ can be constructed so that $\gamma N \backslash\left\{\omega_{1}\right\}$ is a first-countable, zero-dimensional, locally compact, normal, sequentially compact, but not compact space with a countable, discrete, dense subspace.

W. W. Comfort asked, in a private communication, whether or not a separable, sequentially compact, but noncompact space can be constructed without appeal to the continuum hypothesis. We can now answer this affirmatively.

EXAMPLE 1.5. There is a separable, sequentially compact, locally compact, normal space which is not compact. The construction does not depend on $\mathrm{CH}$. However, first-countability is lost. This raises the new

Question. Can a first-countable separable, sequentially compact, but not compact space, be produced without appeal to $\mathrm{CH}$ ?

For any space $X$, let $s X$ be its sequential coreflection, i.e. the same set with the sequentially open sets as topology (see [ $\mathrm{F}_{3}$, Proof of 5.2]). This leads us to

EXAMPLE 1.6. $s \gamma N$ is a separable, sequentially compact Hausdorff space which is not compact.

The continuum hypothesis is not required. However, without it, we cannot be sure the space is regular, i.e. that the point $\omega_{1}$ can be separated from the now closed set $\omega_{1}$. Hence we feel that Example 1.6 is not a satisfactory answer to Comfort's question. With $\mathrm{CH}$, the point $\omega_{1}$ can be made isolated in $s \gamma N$ so that $s \gamma \boldsymbol{N}$ is another space with all the properties of Example 1.4.

With a little care, it can be insured that $s \gamma N$ is not regular yielding

EXAMPLE 1.7. There is a compact Hausdorff space whose sequential coreflection is not even regular. This shows that in general one can expect little preservation of properties under topological coreflections.

Zenor $[Z]$ has introduced a property between countable paracompactness and paracompactness, and has shown that together with Hausdorffness it implies regularity. He gives an example to show that a countably paracompact Hausdorff 
space need not be regular. Example 1.7 shows that this may also be the case even if the space is separable and sequentially compact.

Constructions. We first define recursively a family $\left\{U_{\alpha}\right\}_{\alpha<\omega_{1}}$ of nonempty clopen subsets of $\beta N \backslash N$ such that $U_{\alpha} \varsubsetneqq U_{\beta}$ whenever $\alpha<\beta$. Let $U_{0}$ be any proper nonempty clopen set, and having found suitable $U_{\alpha}$ for each $\alpha<\gamma$, let $F_{\alpha}$ $=(\beta N \mid N) \mid U_{\alpha}$. Since any $G_{\delta}$ formed from nonempty open sets in $\beta \boldsymbol{N} \mid \boldsymbol{N}$ has a nonempty interior [G-J, 65.8, p. 99], let $A$ be a nonempty clopen subset of $\cap\left\{F_{\alpha} \mid \alpha<\gamma\right\}$ and write $A=B \cup C$ with each of $B$ and $C$ nonempty and clopen, and $B \cap C=\varnothing$. Let $U_{\gamma}=(\beta N \mid N) \backslash B$. Clearly $U_{\alpha} \subsetneq U_{\gamma}$ for all $\alpha<\gamma$ and the existence of $B$ insures that the process can continue, i.e. that $F_{\gamma} \neq \varnothing$.

Adopting the same method employed in the usual proof of Urysohn's lemma (see, for example, [V] or [K]), we construct a continuous function from $\beta N \backslash N$ onto $\omega_{1}+1$. The theorem of Magill, previously quoted, now assures the existence of the desired compactification $\gamma \boldsymbol{N}$, with $\gamma \boldsymbol{N} \backslash \boldsymbol{N}$ homeomorphic to $\omega_{1}+1$.

Since $\omega_{1}+1$ can be thought of as a closed nonsequential subspace of $\gamma N(N$ being locally compact), $\gamma N$ cannot be sequential, and so Example 1.1 is complete.

Let $Y=\gamma N \backslash\left\{\omega_{1}\right\}$. Then $\beta Y=\gamma N$ is totally disconnected and hence zerodimensional, being compact [G-J, 16.17, p. 247]. Thus $Y$ is also zero-dimensional [G-J, 16.11, p. 245]. We will use this fact to show that $Y$ is normal.

If $A$ and $B$ are disjoint closed subsets of $Y$, and $A_{1}$ and $B_{1}$ are their respective intersections with $\omega_{1}$ thought of as a subset of $Y$, one of them, say $A_{1}$, is compact [G-J, 5.12(b), p. 74]. Then there is a clopen subset $U$ of $Y$ containing $A_{1}$ and missing $B$. Then $U \cup A$ is a clopen subset of $Y$ containing $A$ and missing $B$. Indeed, points of $A \backslash U$ are isolated, whence $U \cup A$ is open; they can accumulate only in $A_{1} \subseteq U$, whence $U \cup A$ is closed. Thus $Y$ is normal.

If $C$ is any compact open subset of $\omega_{1} \subseteq Y$, then for some compact open $U \subseteq Y, C=U \cap \omega_{1}$. By removing the countably many points of $U \cap N$ one at a time, we see that $C$ is a $G_{\delta}$. But each point of $\omega_{1} \subseteq Y$ is the intersection of countably many such $C$. Hence each point of $Y$ is a $G_{\delta}$. Since $Y$ is locally compact (being an open subset of $\gamma N$ ), it is first countable.

Noting that $Y$ has cardinality $\aleph_{1}$, and that, having $\omega_{1}$ as a closed subset, it is not metacompact completes Example 1.3.

We now turn our attention to $s \gamma N$. Since the cardinality of $\gamma N$ is $\boldsymbol{N}_{1}$ it is sequentially compact $\left[\mathrm{F}_{4}\right.$, Corollary, p. 598]. But $s \gamma N$, having the same convergent sequences, must then also be sequentially compact. Since $\gamma N$ is not sequential, $s \gamma N$ carries a strictly finer topology and is therefore not compact. Thus Example 1.6 is complete.

In the original construction of $\gamma N$ a transfinite sequence $\left\{U_{\alpha}\right\}_{\alpha<\omega_{1}}$ of clopen subsets of $\beta \boldsymbol{N} \backslash \boldsymbol{N}$ was employed. By confining our construction to the complement of some given proper clopen subset $U$, we can assure that $\bigcup\left\{U_{\alpha} \mid \alpha<\omega_{1}\right\}$ is not dense in $\beta N \backslash N$. Every such $U$ is of the form $\left(\operatorname{cl}_{\beta} N A\right) \mid N$ for some subset $A$ of $N$ [G-J, 65.4, p. 99]. Any sequence in $\gamma N$ which is an enumeration of $A$ must converge to $\omega_{1}$. 
Indeed $\omega_{1}$ is its only cluster point by the continuity of the function $\beta N \rightarrow \gamma N$, and hence its limit point, since we are in a compact Hausdorff space.

Now suppose we have separated the point $\omega_{1}$ from the set $\omega_{1}$ by disjoint open subsets $V$ and $W$ of $s \gamma N$. $V$ then must eventually contain any sequence in $N$ converging to the point $\omega_{1}$. Hence $\left(\operatorname{cl}_{\beta N} V \mid\left\{\omega_{1}\right\}\right) \backslash N$ is in fact the complement in $\beta N \mid N$ of the closure of $\bigcup\left\{U_{\alpha} \mid \alpha<\omega_{1}\right\}$. This leaves the closure of that union open, a contradiction [G-J, 6W.3, p. 100]. Thus if some sequence in $N$ converges to the point $\omega_{1}$ in $\gamma \boldsymbol{N}$, then $s \gamma \boldsymbol{N}$ is not regular. This completes Example 1.7.

The continuum hypothesis can be used to assure that no sequence from $N$ converges to $\omega_{1}$. The transfinite recursion used to construct the $\left\{U_{\alpha}\right\}_{\alpha<\omega_{1}}$ can be continued so long as $\bigcup\left\{U_{\alpha}\right\}$ is not dense in $\beta N \mid N$. That $G_{\delta}$ 's in $\beta N \mid N$ have nonempty interiors insures that the process will not terminate for some $\delta<\omega_{1}$. This was the crucial fact of the first construction. However, by cardinality, the process must terminate for some $\delta \geqq \omega_{1}$, i.e. $\bigcup\left\{U_{\alpha}\right\}_{\alpha<\delta}$ is dense in $\beta N \mid \boldsymbol{N}$. Since there are exactly c clopen sets in $\beta N \backslash N$ (again [G-J, 6S.4, p. 99]), and a new one is created for each $\alpha$, the cardinality of $\delta$ is less or equal to $\mathrm{c}$. Assuming $\mathrm{CH}$, the cardinality of $\delta$ is $\aleph_{1}$ and hence there is a cofinal subset of $\delta$ of type $\omega_{1}$ (otherwise $\bigcup\left\{U_{\alpha}\right\}_{\alpha<\delta}$ could not be dense). The $U_{\alpha}$ 's indexed by this subset form a strictly ascending chain of clopen sets indexed by $\omega_{1}$ whose union is dense in $\beta N \backslash N$. If $\gamma N$ is constructed from this chain, then no sequence in $N$ will converge to the point $\omega_{1}$.

In this case, removing $\omega_{1}$ leaves $\gamma N \mid\left\{\omega_{1}\right\}$ sequentially compact (recall that $\gamma N$ is always sequentially compact, regardless of its construction). Since the other properties are independent of the choice of the $U_{\alpha}$ 's this completes Example 1.4. A similar example occurs by taking $s \gamma N$ in this case, since $\omega_{1}$ then becomes isolated (being sequentially open) and hence $s \gamma N$ has the same properties as $\gamma N \mid\left\{\omega_{1}\right\}$.

Having carefully constructed $\gamma \boldsymbol{N}$ so that no sequence in $N$ converges to the point $\omega_{1}$, let $\mathscr{F}$ be the trace on $N$ of the neighborhood filter of $\omega_{1}$ in $\gamma N$. Let $\mathscr{U}$ be any ultrafilter containing $\mathscr{F}$. Let $X$ be a space whose underlying set is $\gamma N$, and in which the neighborhoods of points are as in $\gamma N$, except that a basic neighborhood of the point $\omega_{1}$ is of the form $\left\{\omega_{1}\right\} \cup U$, where $U \in \mathscr{U}$. The space $X$ is clearly sequentially compact due to the careful construction of $\gamma \boldsymbol{N}$.

The space $X$ is not sequential since $\left\{\omega_{1}\right\}$ is sequentially open but not open. It is a $c$-space since each point other than $\omega_{1}$ has a countable neighborhood base, and $\omega_{1}$ has a base of countable neighborhoods. $X$ is clearly Hausdorff, completing Example 1.2 , but fails to be regular since the point $\omega_{1}$ does not have a basis of closed neighborhoods. This leaves the question: Is a sequentially compact regular $c$-space always sequential? An affirmative answer would be enough to restore faith in the now doubtful Theorem $B$ of $\left[F_{4}\right]$.

For Example 1.5, let $\delta$ be as above. Without $\mathrm{CH}$ we can only conclude that $\omega_{1} \leqq \delta \leqq c$ and that $\delta$ has no countable cofinal subset. Defining a function $\beta \boldsymbol{N} \mid \boldsymbol{N} \rightarrow \delta+1$ as before, we obtain a compactification $X^{\prime}$ of $\boldsymbol{N}$ with remainder $X^{\prime} \backslash N=\delta+1$ in the order topology. Since $X^{\prime}$ is totally disconnected and therefore 
zero dimensional, $X=X^{\prime} \mid\{\delta\}$ is also zero dimensional. Since $\delta$ has no countable cofinal subset, of two disjoint closed subsets of $\delta$, one must be compact. These two facts are all that is required to prove that $X$ is normal (see page 308 ).

It remains to show that $X$ is sequentially compact. If we assume that $c<2^{\aleph_{1}}$, this follows as before. However this is only replacing one independent set theoretic assumption $(\mathrm{CH})$ by another, albeit weaker, one $\left(c<2^{\aleph_{1}}\right)$. This can be avoided as follows. Suppose $\left\{x_{n}\right\}$ is any sequence of distinct points in $X$. If $\left\{x_{n}\right\} \cap \delta$ is infinite, $\left\{x_{n}\right\}$ has a convergent subsequence since $\delta$ is sequentially compact. If not, we may assume that $\left\{x_{n}\right\} \subseteq N$. Then $\left\{x_{n}\right\}^{*}=\mathrm{cl}_{\beta} N\left\{x_{n}\right\} \mid\left\{x_{n}\right\}$ must intersect some $U_{\alpha}$ since their union is dense. Now $U_{\alpha}=A_{\alpha}^{*}$ for some $A_{\alpha} \subseteq N$ and $A_{\alpha}^{*} \cap\left\{x_{n}\right\}^{*} \neq \varnothing$ implies that $A_{\alpha} \cap\left\{x_{n}\right\}$ is infinite. This intersection, thought of as a subsequence of $\left\{x_{n}\right\}$, has cluster points, relative to $\beta N$, only in $U_{\alpha}$, and hence has $\alpha$ as its only cluster point relative to $X^{\prime}$. Thus the subsequence converges to $\alpha$ in $X$ and the proof is complete.

We wish to thank W. W. Comfort for several valuable comments concerning this section.

2. An important subclass of the sequential spaces are the Fréchet spaces, i.e. those in which the closure of any set is simply the set of all its sequential limits. Clearly every first countable space (and hence every metric space) is Fréchet. Examples of Fréchet spaces which are not first-countable fairly abound: the reals with the integers identified, the plane with the $X$-axis shrunk to a point, any CW complex which is not locally finite, etc. An example of a sequential space which is not Fréchet can be found in [ $\mathrm{F}_{2}$, Example 2.2].

In 1937 E. Čech asked if there was a Fréchet space (in the convergence space, not the topological, sense (see [N, p. 3]) in which no point had a countable basis of neighborhoods. J. Novak produced such a convergence space $[N, \S 6$, p. 16] which was not a topological convergence, and remarked that he did not know a topological example [N, p. 17].

We now have such an example; it will be presented in $\S 3$. On hearing of our example, Professor Novak informed us that he also had such an example (quite different from ours) which he described in terms of convergence groups of sets, convergence being order convergence relative to inclusion.

This section is devoted to an account of what we believe to be an example which is essentially the same as Novak's, in a different, and more accessible guise, that of zero-one sequences.

EXAMPLE 2.1. There is a zero-dimensional topological group which is a Fréchet space but is not first countable.

Let $2^{\omega_{1}}$ be the topological product of two point discrete spaces $\{0,1\}_{\alpha}$ indexed by the countable ordinals $\omega_{1}$. Let $X$ be the subspace of $2^{\omega_{1}}$ consisting of all functions taking the value 1 at most countably many times.

$X$ as a subspace of $2^{\omega_{1}}$ is a topological group under pointwise addition and hence 
is homogeneous and completely regular. It is also zero dimensional, since $2^{\omega_{1}}$ is. It fails to be locally compact since it is a nonopen dense subset of $2^{\omega_{1}}$.

No point of $X$ is a $G_{\delta}$; thus $X$ is not first countable. Indeed, countably many basic open sets can restrict only countably many coordinates and hence cannot intersect in a point, even in $X$.

If $F \subseteq X$ and $g \in \operatorname{cl} F$, then there is a countable subset $F^{\prime}$ of $F$ with $g \in \operatorname{cl} F^{\prime}$, i.e. $X$ is a $c$-space. Indeed, let $\alpha_{0}=\sup g^{-1}(1)$ and choose a countable subset $F_{0}$ of $F$ containing a function belonging to each of those basic neighborhoods of $g$ which restrict only coordinates $\leqq \alpha_{0}$. Let $\alpha_{1} \geqq \sup \left\{\alpha<\omega_{1} \mid f(\alpha)=1\right.$ for some $\left.f \in F_{0}\right\}$. Choose a countable subset $F_{1}$ of $F$ meeting every basic neighborhood of $g$ restricting only coordinates $\leqq \alpha_{1}$. Having chosen $F_{n}$ similarly for $\alpha_{n}$, let

$$
\alpha_{n+1} \geqq \sup \left\{\alpha<\omega_{1} \mid f(\alpha)=1 \text { for some } f \in F_{n}\right\} .
$$

Thus we construct recursively a sequence of ordinals $\alpha_{0}, \alpha_{1}, \ldots$ (without loss of generality we may take them strictly increasing-we are indebted to F. G. Slaughter, Jr., for this simplification) and a sequence of countable subsets of $F$ having the property that each basic neighborhood of $g$ which restricts no coordinate $\alpha$ strictly between $\alpha_{n}$ and $\alpha_{n+1}$ (i.e. $\alpha_{n}<\alpha \leqq \alpha_{n+1}$ ) meets $F_{n}$. Since each basic neighborhood of $g$ restricts only finitely many coordinates, it leaves some $\left(\alpha_{n}, \alpha_{n+1}\right]$ unrestricted and so meets $F_{n}$. Hence $g \in \mathrm{cl} \bigcup F_{n}$, which is countable.

Finally $X$ is a Fréchet space. Indeed, if $g \in \operatorname{cl} F$, choose a countable subset $F^{\prime}$ of $F$ with $g \in \operatorname{cl} F^{\prime}$. Let $\left\{\alpha_{1}, \alpha_{2}, \ldots\right\}$ be some enumeration of the set of coordinates mapped to 1 either by $g$ or by some $f \in F^{\prime}$. For each $n<\omega_{0}$, let

$$
F_{n}=\left\{f \in F^{\prime} \mid f\left(\alpha_{i}\right)=g\left(\alpha_{i}\right), i \leqq n\right\} .
$$

Then for each $n, g \in \operatorname{cl} F_{n}$ (hence $F_{n} \neq \varnothing$ ) and if we choose $f_{n}$ arbitrarily from $F_{n},\left\{f_{n}\right\}$ will converge (pointwise) to $f$. This completes the proof.

The authors are indebted to M. Venkataraman for suggesting that Novak's example might be recast in this simple way, and to T. Soundararajan for a key idea in the original proof.

It has recently come to our attention that an as yet unpublished result of Noble $\left[\mathrm{N}_{1}\right]$ considerably generalizes Example 2.1. A $\Sigma$-subspace of a product space is one consisting of all those functions agreeing with a given fixed function except at countably many indexes. Noble proves $\left[\mathrm{N}_{1}\right.$, Theorem 2.1] that any $\Sigma$-subspace of a product of first countable spaces is Fréchet. Example 2.1 is an immediate specialization.

3. Since every first-countable space with unique sequential limits is Hausdorff, and every first-countable space is Fréchet, it is natural to ask whether every Fréchet space with unique sequential limits is Hausdorff. Several examples have been given to show this is not the case (see for example $\left[F_{1}\right]$ ).

Again it was E. Čech who asked whether there was a Fréchet space with unique sequential limits in which no pair of points have disjoint neighborhoods. 
In this section, we propose to give a totally different (and countable) solution to the problem of $\S 2$, and then to give a partial answer to the problem of Čech mentioned in the preceding paragraph.

The method employed in both cases is that of attaching of spaces, which can be traced from Urysohn [U] to Hewitt $[\mathrm{H}]$ to Shimrat's homogeneous extension [S] to the more recent applications in $[\mathrm{A}-\mathrm{F}]$ and $\left[\mathrm{F}_{5}\right]$. The construction of $S_{\omega}$ in $[\mathrm{A}-\mathrm{F}]$ provides a good warm-up for the examples of this section.

EXAMPLE 3.1. There is a countable, zero-dimensional, Hausdorff, homogeneous, Fréchet space which is not first countable.

Note that this space, being $\sigma$-compact and regular, has many nice topological properties, i.e. paracompactness, etc. It fails to be locally compact, as did Example 2.1. It would be interesting to know if a homogeneous Fréchet space could also be locally compact without being first countable.

EXAMPLE 3.2. There is a countable, homogeneous, sequential space with unique sequential limits in which no pair of distinct points have disjoint neighborhoods.

Since every Fréchet space is sequential, this may be considered as partial solution to Čech's problem.

Construction of 3.1. We begin by constructing a sequential fan $F$. Take denumerably many copies of a convergent sequence together with its limit point (i.e. copies of $\{1 / n \mid n \in N\} \cup\{0\}$ as subsets of the real line) and identify the limit points, calling the new point 0 and the new set $F$. Provide $F$ with the quotient topology after having taken a disjoint topological sum of the convergent sequences with limits. The resulting sequential fan $F$ has each of its countably many points isolated except for 0 , which fails to have a countable basis of neighborhoods. The space $F$, however, is certainly a zero-dimensional, Hausdorff, Fréchet space. (Some of these assertions can be most quickly verified by recognizing $F$ as a closed subset of the real line with the integers identified.)

We now begin the attaching process in earnest. Let $F_{1}=F$ and construct $F_{2}$ by attaching to each isolated point $x$ of $F_{1}$ a copy $F^{x}$ of $F$ (all these various copies being kept scrupulously disjoint before attaching) identifying $x$ with the zero $0^{x}$ of $F^{x}$. For a topology, each isolated point of each $F^{x}$ will remain isolated in $F_{2}$; a basic $F_{2}$-neighborhood of $0^{x}$ will be simply a basic $F^{x}$-neighborhood considered as a subset of $F_{2}$; for a basic $F_{2}$-neighborhood of $0\left(\in F_{1}\right)$ in $F_{2}$, choose an $F_{1}$ neighborhood $U$ of 0 and take $U \cup \cup\left\{F^{x} \mid x \in U\right\}$ with the appropriate identifications. Clearly $F_{1}$ is a closed subspace of $F_{2}, F_{2}$ is a Fréchet space, and most importantly, no point in $F_{1}$ has a countable basis of neighborhoods in $F_{2}$.

We proceed by recursion. Having constructed $F_{n-1}$, a copy of $F$ is attached at each isolated point of $F_{n-1}$ to arrive at $F_{n}$. If $F^{x}$ is attached at $x$ we will say that $y \leqq x$ for each $y \in F^{x}$. This relation is defined at each stage of the construction; its transitive closure is a partial order on $F_{n}$. Define the rank of a point $p$ in $F_{n}$ as 0 for $p=0$ in $F_{1}$, and as the least $i$ such that $p \in F_{i}$ otherwise. For $p \in F_{n}$ of rank $n$, let $p$ be isolated. Otherwise, let $U$ be a neighborhood of $p$ in $F^{p}\left(\right.$ take $\left.F^{0}=F_{1}\right)$. Then 
the sets $U^{*}=\left\{y \in F_{n} \mid y \leqq x\right.$ for some $\left.x \in U\right\}$ will form a neighborhood basis for $p$. Again each $F_{i}$ for $i<n$ is a closed subspace of $F_{n}, F_{n}$ is a Fréchet space (this requires a little thought), and no point of rank $<n$ has a countable basis of neighborhoods in $F_{n}$.

Now let $F_{\omega}=\bigcup F_{n}$. We may either take the inductive limit topology (the $F_{n}$ 's together with their inclusion maps form an inductive system; $F_{\omega}$ is the limit) or, preferably, we may extend the partial order to $F_{\omega}$ and use the $U^{*}$ as basic neighborhoods as before. It amounts to the same thing. Each $F_{n}$ is a closed subspace of $F_{\omega}, F_{\omega}$ is Fréchet and no point of $F_{\omega}$ has a countable basis of neighborhoods.

It is obvious that $F_{\omega}$ is Hausdorff. Each $U^{*}$ is clopen (recall that $U$ is a neighborhood of $p$ in some $F^{p}$ ); whence $F_{\omega}$ is zero dimensional. For each $p \in F_{\omega}$ let $I(p)$ be the principal ideal generated by $p$, i.e. $I(p)=\left\{y \in F_{\omega} \mid y \leqq p\right\}$. Then each $I(p)$ and each $F_{\omega} \backslash I(p)$ is homeomorphic to $F_{\omega}$. Homogeneity follows easily by finite induction. This completes Example 3.1.

It would be interesting to know if $F_{\omega}$ can support a group structure as does Example 2.1.

One should note that having specified any infinite cardinal $\mathfrak{m}$, an example similar to $F_{\omega}$ can be constructed having all the same properties (except countability) with the character of each point $\geqq \mathfrak{m}$. One simply must put more sequences in the fan.

If one wanted only a sequential space instead of a Fréchet space in Example 3.1, it could be had simply by taking Shimrat's homogeneous extension of $F$. The Fréchet property is lost through quotients generally, where sequentialness is not. The space in this case would apparently be much more complicated as a set than $F_{\omega}$.

Construction of 3.2. Example 3.2 is significantly more complex than is Example 3.1. This is so on two counts: first the basic building block is more complicated; secondly, the attaching is performed at two points each time instead of at one, and this is done for "almost all" pairs of points.

The basic building block $D$ is sort of a "sequential fan with two pivots". Precisely, $D$ consists of an infinite sequence $B_{1}, B_{2}, \ldots$ of pairwise disjoint countably infinite sets of isolated points ( $B_{n}$ is called the $n$th blade) together with two additional distinct points 0 and $0^{\prime}$ whose neighborhoods are described as follows. To form a basic neighborhood of 0 , one may discard finitely many points (including possibly zero) from each even numbered blade, as well as finitely many odd numbered blades in their entirety; the basic neighborhoods of $0^{\prime}$ are formed similarly with odd and even interchanged. The resulting countable space is $D$.

It is clear from the definition that 0 and $0^{\prime}$ have no disjoint pair of neighborhoods. If 0 is in the closure of some subset $A$ of $D$, then either $A \cap B_{n}$ is infinite for some even $n$, in which case any enumeration of $A \cap B_{n}$ is a sequence in $A$ converging to 0 , or $A$ has a nonempty intersection with infinitely many blades of odd index, in which case a point chosen arbitrarily from each of these intersections gives rise again to a sequence in $A$ converging to 0 . Using the dual (in the sense of odd and 
even) we conclude that $D$ is a Fréchet space. Any sequence in $D \backslash\{0\}$ converging to 0 must be either infinitely many times in some even numbered blade or else only finitely many times in each of infinitely many odd numbered blades. In any event, it cannot also converge to $0^{\prime}$. Hence sequential limits are unique and we have another example such as was mentioned in the first paragraph of this section, i.e. a nonHausdorff, Fréchet space with unique sequential limits.

The non-Hausdorffness occurs only at the points 0 and $0^{\prime}$. We will use the attaching process to construct a space $D_{\omega}$ in which every pair of distinct points looks like a complicated version of the pair $0,0^{\prime}$.

Let $D_{0}$ be the two point discrete space $\{a, b\}$. To get $D_{1}$, simply attach a copy of $D$ to $D_{0}$ identifying 0 with $a$ and $0^{\prime}$ with $b$. We will say that the pair of distinct points are joined if they have been identified with the points 0 and $0^{\prime}$ of some copy of $D$. Thus $a$ and $b$ are joined in $D_{1}$. To get $D_{2}$, to each pair of distinct nonjoined points of $D_{1}$ attach a copy of $D$. In general, having constructed $D_{n}$, for each pair $\{x, y\}$ of nonjoined points of $D_{n}$ choose a copy $D^{\{x, y\}}$ of $D$ and attach it at $x$ and $y$, thus arriving at $D_{n+1}$. Each $D_{n}$ is a proper subset of $D_{n+1}$. Let $D_{\omega}$ be the union of all the $D_{n}$.

We topologize $D_{\omega}$ a bit at a time. Having given $D_{0}$ the discrete topology and each copy $D^{\{x, y\}}$ of $D$ the topology described above for $D$, each $D_{n+1}$ can be regarded as a quotient of the disjoint topological sum of $D_{n}$ and countably many copies of $D$. Give $D_{n}$ the quotient topology. As before, $D_{\omega}$ can be regarded as the inductive limit of an inductive system composed of the $D_{n}$ and compositions of their inclusion maps into each other. Give $D_{\omega}$ the inductive limit topology.

Clearly $D_{\omega}$ is sequential (since we began with sequential spaces and essentially performed only sums and quotients (see $\left.\left[\mathrm{F}_{2}, 1.2,1.6,1.7\right]\right)$ ). It is also clear that no pair of distinct points of $D_{\omega}$ have disjoint neighborhoods. It remains only to show that sequential limits are unique. This becomes clear once one realizes that a sequence can converge to a point $x$ only if $A$ is eventually in some finite number of $D^{\{x, y\}}$.

Unfortunately $D_{\omega}$ is not a Fréchet space, so that the problem of Čech remains open. One might be tempted to redefine the topology of $D_{\omega}$ in a manner more analogous to that of $F_{\omega}$ in order to make $D_{\omega}$ Fréchet. This can surely be done, but the uniqueness of sequential limits is lost in the process.

\section{REFERENCES}

[A-F] A. V. Arhangel'skiǐ and S. P. Franklin, Ordinal invariants for topological spaces, Michigan Math. J. 15 (1968), 313-320, 506. MR 39 \#2112.

$\left[F_{1}\right]$ S. P. Franklin, On unique sequential limits, Nieuw Arch. Wisk. (3) 14 (1966), 12-14. MR 33 \#690.

$\left[\mathrm{F}_{2}\right]-$, Spaces in which sequences suffice, Fund. Math. 57 (1965), 107-115. MR 31 \#5184.

$\left[\mathrm{F}_{3}\right]-$, Spaces in which sequences suffice. II, Fund. Math. 61 (1967), 51-56. MR 36 \#5882. 
[F $\left.\mathrm{F}_{4}\right]$ S. P. Franklin, On two questions of Moore and Mrowka, Proc. Amer. Math. Soc. 21 (1969), 597-599.

$\left[\mathrm{F}_{5}\right] \longrightarrow$, A homogeneous Hausdorff $E_{0}$-space which isn't $E_{1}$, General Topology and its Relations to Modern Analysis and Algebra, III, Proc. Kanpur Top. Conf. (to appear).

[G-J] L. Gillman and M. Jerison, Rings of continuous functions, University Series in Higher Math., Van Nostrand, Princeton, N. J., 1960. MR 22 \#6994.

[H] E. Hewitt, On two problems of Urysohn, Ann. of Math. (2) 47 (1946), 503-509. MR 8, 165.

[K] J. L. Kelley, General topology, Van Nostrand, Princeton, N. J., 1955. MR 16, 1136.

[M] K. D. Magill, Jr., A note on compactifications, Math. Z. 94 (1966), 322-325. MR 34 \#3530.

[McA] L. F. McAuley, Paracompactness and an example due to F. B. Jones, Proc. Amer. Math. Soc. 7 (1956), 1155-1156. MR 18, 496.

[N] J. Novak, Sur les expaces $(\mathscr{L})$ et sur les produits cartésiens $(\mathscr{L})$, Publ. Fac. Sci. Univ. Masaryk No. 273 (1939). MR 1, 221

[ $\left.\mathrm{N}_{1}\right] \mathrm{N}$. Noble, The continuity of functions on cartesian products, Trans. Amer. Math. Soc. 149 (1970), 187-198.

[P] I. I. Parovičenko, On a universal bicompactum of weight $\aleph$, Dokl. Akad. Nauk SSSR 150 (1963), 36-39=Soviet Math. Dokl. 4 (1963), 592-595. MR 27 \#719.

$\left[\mathrm{R}_{1}\right]$ M. E. Rudin, A separable normal nonparacompact space, Proc. Amer. Math. Soc. 7 (1956), 940-941. MR 18, 429.

$\left[\mathrm{R}_{2}\right] \longrightarrow$, A technique for constructing examples, Proc. Amer. Math. Soc. 16 (1965), 1320-1323. MR 33 \#6403.

[S] M. Shimrat, Embedding in homogeneous spaces, Quart. J. Math. Oxford Ser. (2) 5 (1954), 304-311. MR 16, 845.

[U] P. Urysohn, Über die Mächtigkeit der zusammenhängenden Mengen, Math. Ann. 94 (1925), 262-295.

[V] R. Vaidyanathaswamy, Set topology, 2nd ed., Chelsea, New York, 1960. MR 22 \#5953.

[Z] P. Zenor, A class of countably paracompact spaces, Proc. Amer. Math. Soc. 24 (1970), 258-262.

Carnegie-Mellon Univeristy,

Pittsburgh, Pennsylvania 15213

INDIAN INSTITUTE OF TECHNOLOGY,

KANPUR, INDIA

UNIVERSITY OF ILLINOIS,

URBANA, ILLINOIS 61801

UNIVERSITY OF MADURAI,

MADRAS, INDIA 